\title{
Analysis and Identification of two Soaps by Infrared Absorption Spectra and Reflectance Graphs
}

\section{ISSN : 2688-8394}

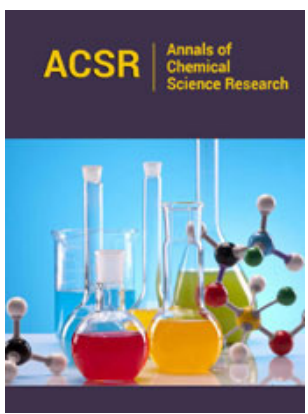

*Corresponding author: Chryssou K, General Chemical State Laboratory, Greece

Submission: 钲 December 02, 2019

Published: 制December 09, 2019

Volume 1 - Issue 5

How to cite this article: Chryssou $\mathrm{K}$, Stassinopoulou M, Lampi E. Analysis and Identification of two Soaps by Infrared Absorption Spectra and Reflectance Graphs. Ann Chem Sci Res. 1(5). ACSR.000523.2019.

DOI: 10.31031/ACSR.2019.01.000523

Copyright@ Chryssou K, This article is distributed under the terms of the Creative Commons Attribution 4.0 International License, which permits unrestricted use and redistribution provided that the original author and source are credited.

\author{
Chryssou K*, Stassinopoulou M and Lampi E \\ General Chemical State Laboratory, Greece
}

\begin{abstract}
Two soap bars one green and another white green were analyzed, and their total fatty matter content, unsaponifiable, un-saponified and un-saponified saponifiable matter content, ethanol-insoluble matter, and free caustic alkali content were determined. The infrared spectra of total lipids, unsaponifiable fats, and insoluble matter in ethanol, were recorded for the two soaps. The Kubelka-Munk K/S values were calculated using the expression depended on reflectivity $\mathrm{R}$ of the observed soap's surface, as well as the CIE L*a*b* values for both soaps.
\end{abstract}

Keywords: Soap; Total fatty matter content; FT IR spectra; Kubelka-Munk expression; Color in the CIE L*a*b* system

\section{Introduction}

Chemically soap is a mixture of the sodium or potassium salts of long chain fatty acids produced by hydrolysis (saponification) of fat with alkali. Wood ash was used before as a source of alkali, when $\mathrm{NaOH}$ became commercially available. Crude soap curd contains glycerol and excess alkali as well as soap, and purification is affected by boiling with a large amount of water and adding $\mathrm{NaCl}$ to precipitate the pure sodium carboxylate salts. The smooth soap that precipitates is dried, perfumed and pressed into bars for household use. Soaps act as cleansers because the two ends of a soap molecule are so different. The sodium salt end of the long-chain molecule is ionic and therefore hydrophilic; it tries to dissolve in water. The long hydrocarbon chain portion of the molecule however is nonpolar and lipophilic; it tries to dissolve in grease. The net effect of these two opposing tendencies is that soaps are attracted to both grease and water and are therefore valuable as cleansers.

When the soap is dispersed in water the long hydrocarbon tails cluster together in a lipophilic ball, while the ionic heads on the surface of the cluster stick out into the water layer. These spherical clusters are called micelles. Grease and oil droplets are solubilized in water when they are coated by the nonpolar tails of the soap molecules in the center of micelles. Once solubilized the grease and dirt can be rinsed away. In this work the two soaps were analyzed and characterized as finished products. The green and white-green soaps are generally considered as anionic surface-active agents [1,2].

\section{Experimental}

\section{Reagents}

a. Ethanol absolute anhydrous (1L) Carlo Erba UN1170 Gradient grade

b. Ethanol $95 \% \mathrm{v} / \mathrm{v}$ solution, neutralized to the phenolphthalein solution

c. Sulphuric acid RPE 0.5M (1N) For analysis Carlo Erba UN 2796

d. Sodium hydroxide 0.1N (N/10) RPE UN 1824 For analysis Carlo Erba

e. Potassium hydroxide approximately $0.5 \mathrm{~N}$ standard volumetric solution in ethanol 
f. KOH (Potassium hydroxide) 1Kg ERFAR, MW:56,11, DAB 6, B.P. 1968

g. Methyl orange $0.2 \% \mathrm{w} / \mathrm{w}$

h. Phenolphthalein $1 \% \mathrm{w} / \mathrm{v}$ in ethanol $95^{\circ}$

i. Light petroleum ether, boiling range between 40-60oC RPE for analysis Carlo Erba UN 1268

\section{Apparatus}

A. pH-meter Metrohm 716 DMS Titrino, Swiss made

B. Analytical balance Mettler Toledo AB 204-S/FACT accurate to $0,1 \mathrm{mg}$, maximum capacity $220 \mathrm{~g}$

C. Oven Memmert direkt, capable of being controlled at $103{ }^{\circ} \mathrm{C} \pm 2$ ${ }^{0} \mathrm{C}$

D. Water bath FALC, $220 / 240 \mathrm{~V}, 50 \mathrm{~Hz}$

E. FT-IR Spectrometer Perkin Elmer Inc Spectrum 2000 Version 5.0.2 Copyright 2004

F. Spectrophotometer CM-3630 BCMTS M Type 40605, S.N. 43029, Touch-Screen-M V 2.0, Frank-PTI

Ordinary laboratory apparatus: Beaker of capacity $250 \mathrm{ml}$, squat form, three separating funnels capacity $500 \mathrm{ml}$.

Sample preparation: The samples of the two soaps were analyzed directly and no sample preparation was required on the finished product, green soap, and white soap.

\section{Analysis of soaps}

\section{Procedure}

Determination of the total fatty matter content of the soap: We weighed $5.04 \mathrm{~g}$ of the green soap into a $250 \mathrm{ml}$ beaker, and $5.07 \mathrm{~g}$ of the second green-white soap into a $250 \mathrm{ml}$ second beaker. We then dissolved the test portions in $100 \mathrm{ml}$ of hot water and we poured the solutions into one of three separating funnels. We washed the beaker with small quantities of water nearly $50 \mathrm{ml}$ and we added the washings to the first separating funnel. We added a few drops of a methyl orange solution and then from a burette we added, while vigorously shaking, the separating funnel, $20 \mathrm{ml}$ of a $1 \mathrm{~N}$ sulphuric acid solution until the color of the solution changed from yellow to pink. We then cooled the contents of the separating funnel to about $25^{\circ} \mathrm{C}$ and we added $100 \mathrm{ml}$ of light petroleum ether $40-60{ }^{\circ} \mathrm{C}$. We inserted the stopper and we gently inverted the separating funnel whilst maintaining a hold on the stopper [3-5]. We opened the stopcock of the separating funnel gradually to release the pressure, then we closed it and we gently shacked and again released the pressure. We repeated the shaking until the aqueous layer became clear and we then allowed to stand. Then we ran off the aqueous layer into the second separating funnel and we extracted with $100 \mathrm{ml}$ of the light petroleum ether. We repeated the process of the extraction with $50 \mathrm{ml}$ of the light petroleum ether into a third separating funnel and we then collected the aqueous layer in a conical flask, and we combined the three light petroleum extracts in the first separating funnel. We then washed the light petroleum extract by shaking with $30 \mathrm{ml}$ water until the washings were neutral to the methyl orange solution. We carried out three washings with $30 \mathrm{ml}$ of water. We allowed each wash to stand for at least $5 \mathrm{~min}$ to give a clear line of demarcation between the layers and we then ran off the aqueous layer. We evaporated off all of the washed light petroleum solutions in a glass container on the water bath. We then heated the container with the total fatty matter to constant mass in the oven controlled at $103{ }^{\circ} \mathrm{C} \pm 2{ }^{\circ} \mathrm{C}$ for $1 \mathrm{hr}$. We finally cooled in a desiccator for $40 \mathrm{~min}$, and we weighed the contents.

Yield: $4.21 \mathrm{~g}$ of total fatty matter for the green soap, $83.5 \% \mathrm{w} / \mathrm{w}$, and 3.68g of total fatty matter for the white-green soap, $72.6 \% \mathrm{w} / \mathrm{w}$

Determination of unsaponifiable, un-saponified and unsaponified saponifiable matter: The above residue of the total fatty matter in the glass container was re-dissolved with $50 \mathrm{ml}$ of Ethanol $95 \% \mathrm{v} / \mathrm{v}$ solution on the water bath. It was transferred into the first separating funnel where Phenolphthalein $1 \% \mathrm{w} / \mathrm{v}$ in ethanol $95^{\circ}$ was added. Then approximately $20 \mathrm{ml}$ of the potassium hydroxide approximately $0,5 \mathrm{~N}$ standard volumetric solution in ethanol, were added until the color of the solution changed from yellow to purple. Finally, $5 \mathrm{ml}$ of water were added to facilitate the separation of the two layers. We then extracted with $100 \mathrm{ml}$ of the light petroleum ether. We repeated the process of the extraction with $50 \mathrm{ml}$ of the light petroleum ether into a second separating funnel. The extraction was repeated with another $50 \mathrm{ml}$ of light petroleum ether into a third separating funnel. We then collected the aqueous layer in a conical flask, and we combined the three light petroleum extracts in the first separating funnel. We then washed the light petroleum extract by shaking with $20 \mathrm{ml}$ of ethanol solution $58^{\circ}$ until the washings were clear. We carried out five washings with $20 \mathrm{ml}$ of the ethanol solution $58^{\circ}$. We allowed each wash to stand for at least $5 \mathrm{~min}$ to give a clear line of demarcation between the layers and we then ran off the alcoholic layer. We evaporated off all of the washed light petroleum solution in a glass container on the water bath. We then heated the container with the unsaponifiable, unsaponified and un-saponified saponifiable matter to constant mass in the oven controlled at $103^{\circ} \mathrm{C} \pm 2{ }^{\circ} \mathrm{C}$, for $1 \mathrm{hr}$. We finally cooled in a desiccator for $40 \mathrm{~min}$, and we weighed the contents.

Yield: $0.05 \mathrm{~g}, 1.0 \% \mathrm{w} / \mathrm{w}$ for the green soap, and $0.17 \mathrm{~g}, 3.4 \% \mathrm{w} / \mathrm{w}$ for the white-green soap.

Determination of content of ethanol-insoluble matter: We weighed $5.00 \mathrm{~g}$ of the green soap into a $250 \mathrm{ml}$ beaker, and $5.14 \mathrm{~g}$ of the white soap into another $250 \mathrm{ml}$ beaker. We added $150 \mathrm{ml}$ of the ethanol $95 \% \mathrm{v} / \mathrm{v}$ in the beakers and heated on the water bath to gentle boiling. We have dried before the filter papers to be used for the filtration of the insoluble matter in the oven controlled to 103 ${ }^{\circ} \mathrm{C}$, for an hour. We allowed to the filter papers to cool to ambient temperature in a desiccator for $20 \mathrm{~min}$, and we weighed them to be $0.99 \mathrm{~g}$ and $1.08 \mathrm{~g}$ respectively for each soap. We then placed them in funnels mounted on conical flasks placed on the water bath. When the soap was completely dissolved we decanted the supernatant liquid on the filter paper and then washed the insoluble matter in the conical flask by decantation with ethanol previously heated to near its boiling point and we transferred the insoluble matter to 
the filter paper with small quantities of warm ethanol. The filter paper and the residue were washed with the warm ethanol until entirely free from the soap. During the operation described above the conical flask carrying the funnel was placed on the water bath and the filtrate was kept gently boiling. The funnel was covered with a watch glass so that the wash liquor was not cooled of and the ethanol vapor which condensed on the watch glass and dropped back on the filter paper completed the washing of the latter. We finally dried the filter paper in air, and we placed it in the oven at $103^{\circ} \mathrm{C}$. After $1 \mathrm{~h}$, we removed the filter paper and left it in the desiccator long enough for it to cool completely to ambient temperature, nearly $20 \mathrm{~min}$, and we weighed it.

Yield: $0.02 \mathrm{~g}, 0.4 \% \mathrm{w} / \mathrm{w}$ for the green soap, and $0.0002 \mathrm{~g}$, $0.004 \% \mathrm{w} / \mathrm{w}$ for the white-green soap

Determination of free caustic alkali: In the ethanol solution of the above filtrates were added four (4) drops of phenolophthalein indicator and they were not colored pink but remained colorless. The free caustic alkali was expressed as sodium hydroxide for these two sodium soaps.

The yield was lower than $0.01 \% \mathrm{w} / \mathrm{w} \mathrm{NaOH}$ for both soaps.

\section{Results and Discussion}

\section{Determination of $\mathrm{pH}$}

We prepared a $1 \% \mathrm{w} / \mathrm{w}$ solution of the green soap in deionized water and we measured $\mathrm{pH}=10,2$ at a temperature of $25.7^{\circ} \mathrm{C}$ in the Metro hm 716 DMS Titrino pH-meter. We then prepared a 1\%w/w solution of the white-green soap in water and we measured $\mathrm{pH}=10.3$ at a temperature of $24.4{ }^{\circ} \mathrm{C}$ in the Metrohm 716DMS Titrino $\mathrm{pH}-$ meter.

\section{Acquisition of IR-spectra}

Infrared transmittance data: The infrared spectra were recorded in the solid state. The $\alpha, \beta$-unsaturated and aryl conjugated acids show absorption of the dimer in the $1708 \mathrm{~cm}^{-1}$ peak (Figure 1). In the above spectrum (Figure 2 ) a strong $\mathrm{C}=0$ stretching absorption band in the peak $1558 \mathrm{~cm}^{-1}$ is due to carboxylic acids, carboxylic esters. Its relatively constant position, high intensity, and relative freedom from interfering bands make this one of the easiest bands to recognize in IR spectra of soaps. The $\mathrm{C}=0$ absorption band of saturated aliphatic esters is in the peak $1736 \mathrm{~cm}^{-1}$ (Figure 3).

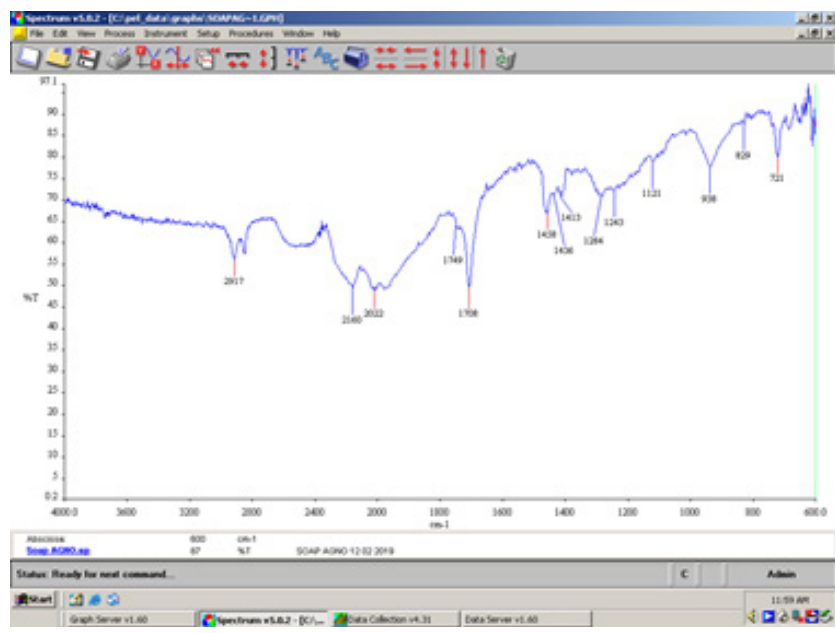

Figure 1: FT-IR spectrum of total lipids in the green soap bar as fatty acids.

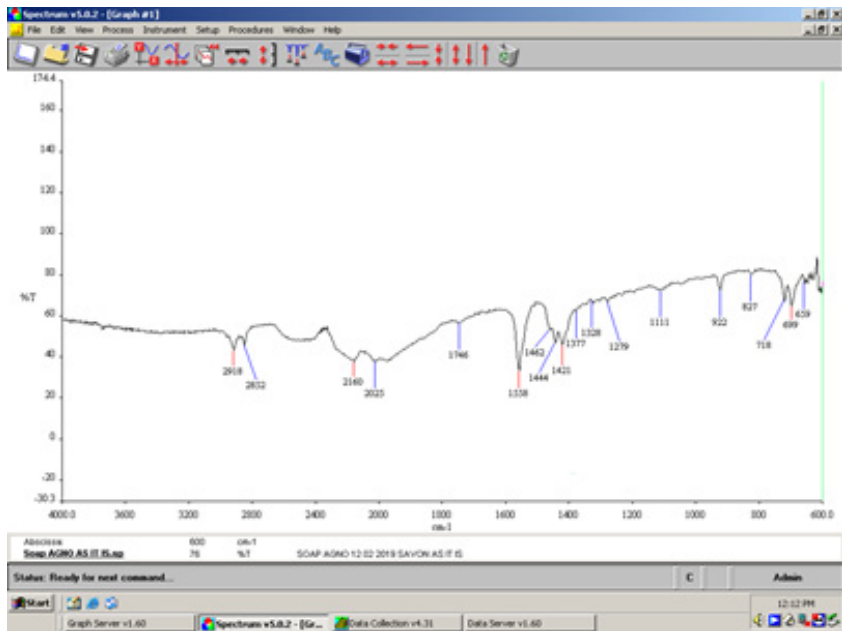

Figure 2: FT-IR spectrum of the green soap bar as it is. 


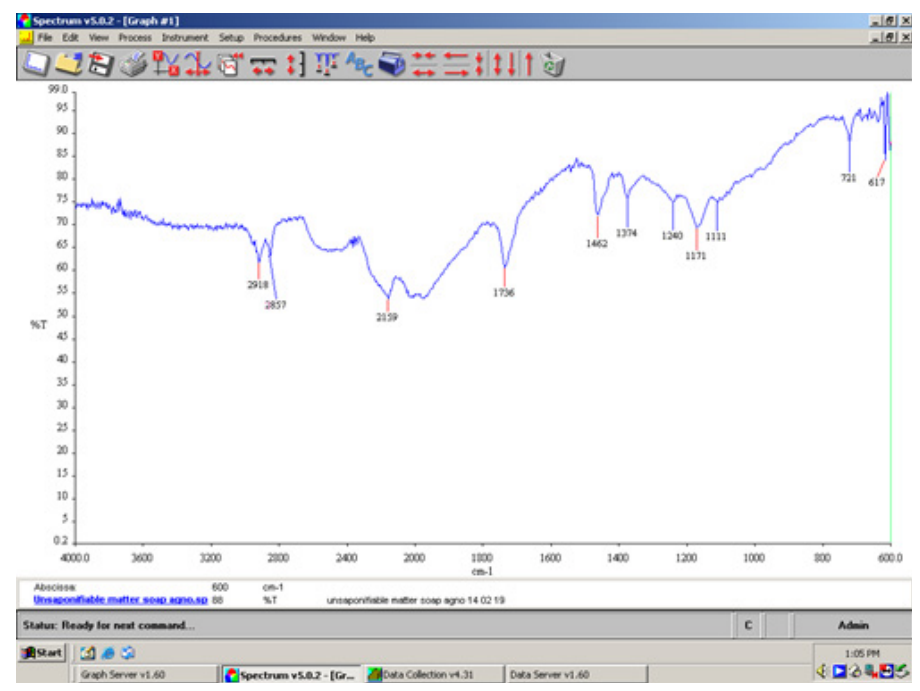

Figure 3: FT-IR spectrum of unsaponifiable fats in the green soap.

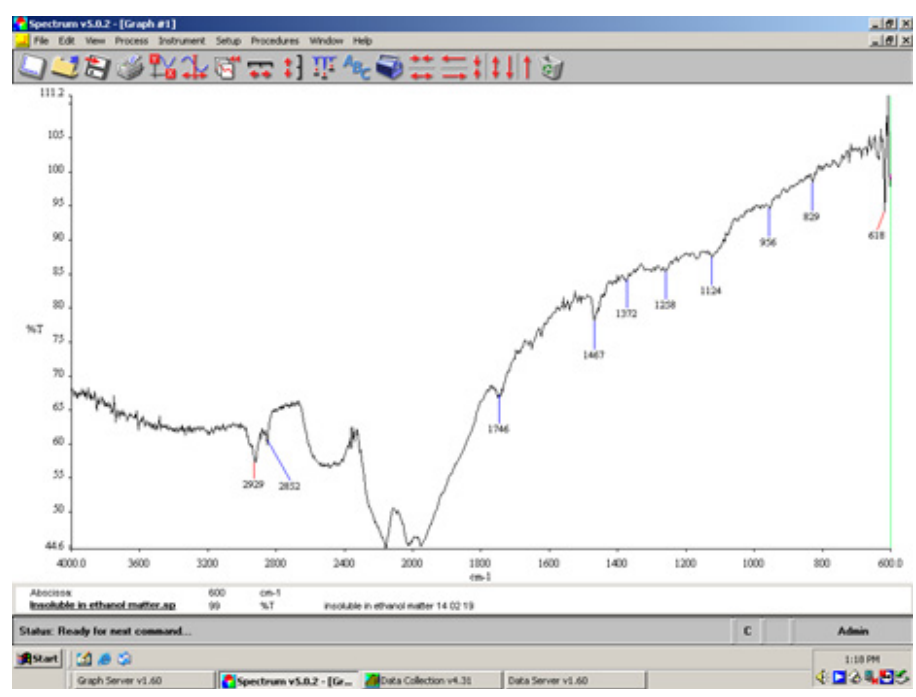

Figure 4: FT-IR spectrum of insoluble matter in ethanol for the green soap indicating sodium carbonate salt in the peak at $1467 \mathrm{~cm}-1$.

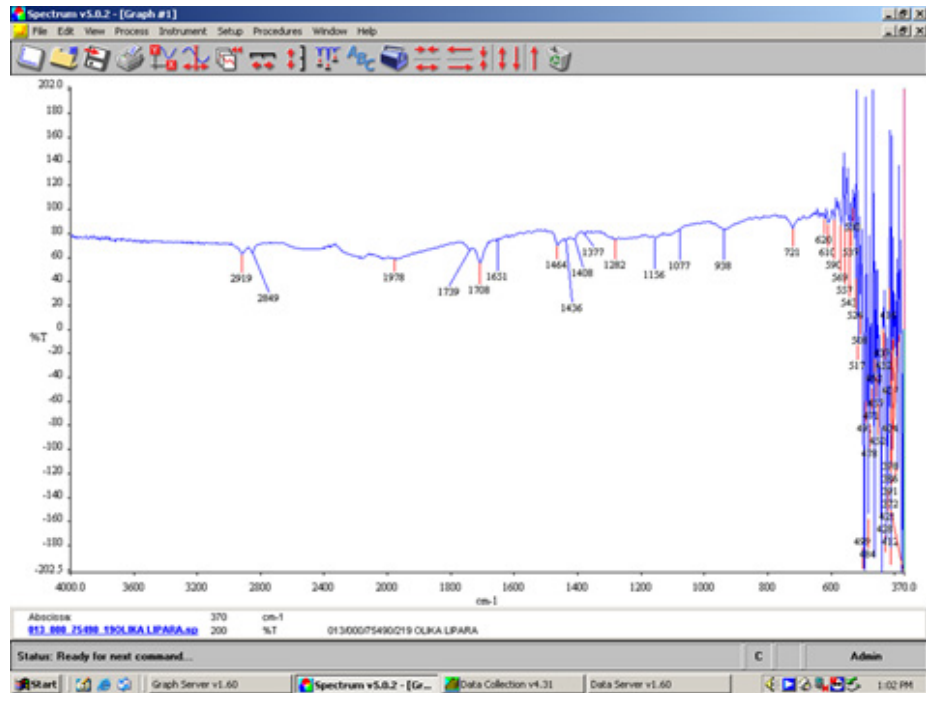

Figure 5: FT-IR spectrum of total lipids in a white-green soap bar. 


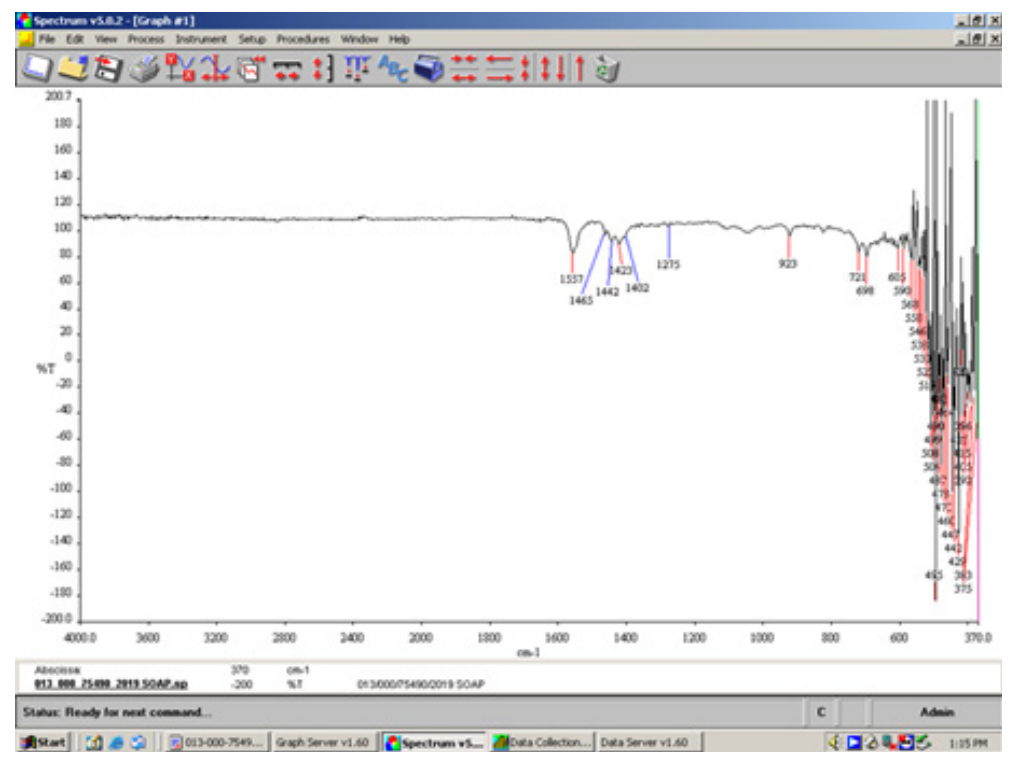

Figure 6: FT-IR spectrum of the white-green soap bar as it is.

In the above spectrum (Figure 4) we identified the sodium carbonate salt. This is because free alkali in soaps usually consists of hydroxide and carbonate of sodium. The free caustic alkali is the hydroxide and is normally expressed as $\mathrm{Na}_{2} \mathrm{O}$. The above method (2.5.1.4) of determination of free caustic alkali can only be applied to pure soaps made from olive oil (Figure 5 ). The $\alpha, \beta$-unsaturated and aryl conjugated acids show absorption of the dimer in the $1708 \mathrm{~cm}^{-1}$ peak. In the above spectrum a strong $\mathrm{C}=0$ stretching absorption band in the peak $1557 \mathrm{~cm}^{-1}$ is due to carboxylic acids, and carboxylic esters present in the soap bar (Figure 6).

The $\mathrm{C}=\mathrm{O}$ absorption band of saturated aliphatic esters appears in the $1738 \mathrm{~cm}^{-1}$ peak. The shifting of the $\mathrm{C}=0$ absorbance band of unsaponifiable fats in the two soaps is due to changes in the structural features of the respective molecules, which follows the degree to which the molecules absorb radiation is also affected (Figure 7).

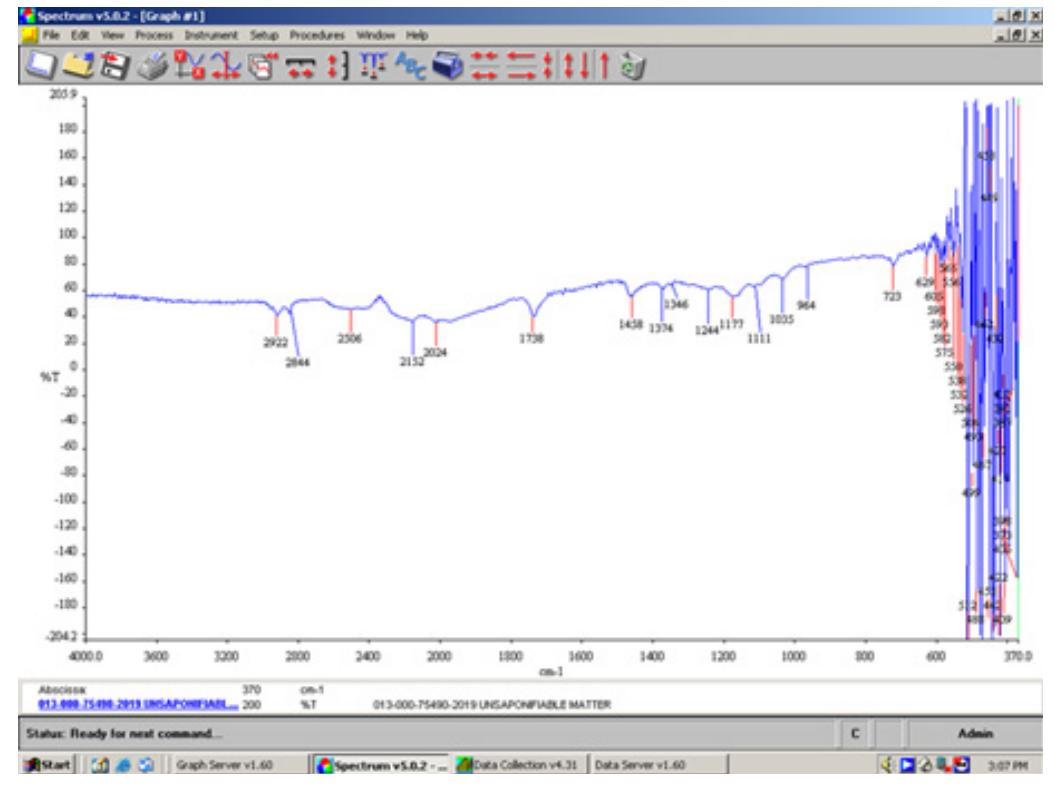

Figure 7: FT-IR spectrum of unsaponifiable fats in the white-green soap.

Calculation of Kubelka-Munk K/S values for the two soaps (absorption coefficient/ scattering coefficient ratio)

The K/S ratio for the green soap was found $\mathrm{K} / \mathrm{S}=11.51$ and the reflectance ISO brightness used was measured $24.98 \%$. The ISO brightness measured is the numerical value of the reflectance of the soap bar at $457 \mathrm{~nm}$, blue light reflectance. The $\mathrm{K} / \mathrm{S}$ ratio for the second soap white green was found $\mathrm{K} / \mathrm{S}=14.92$ and the reflectance ISO brightness used was measured $31.81 \%$. The ISO brightness measured is the numerical value of the reflectance of the soap bar at $457 \mathrm{~nm}$, blue light reflectance. 
In this work the Kubelka-Munk theory is used for predicting optical properties for soaps. The appearance of a soap bar is the result of its optical properties. As known the Kubelka-Munk theory is based on the assumption that the interaction between the diffuse light and the soap material can be described in terms of two fundamental optical constants. The specific scattering coefficient (S) and the specific absorption coefficient (K). Although the KubelkaMunk theory holds strictly for homogeneous materials only, it works also for soap bars containing more than one substance. The equation of Kubelka-Munk used above is: $K / S=(1-R) 2 / 2 R$ where $K$ is the absorption or coefficient of reflectivity and $\mathrm{S}$ is the coefficient of light scattering; $\mathrm{R}$ is the observed reflectivity for monochromatic light (Table 1). From the two K/S ratios calculated above we can assume that light absorption is higher in the first green soap indicating the presence of more colored matter other than soap which is converted to heat thereby reducing the brightness of the green soap bar.

Table 1: Substrate details.

\begin{tabular}{|c|c|c|}
\hline Property & Green Soap & $\begin{array}{c}\text { White-Green } \\
\text { Soap }\end{array}$ \\
\hline ISO Brightness (ISO 2470-1): & $24.98 \%$ & $31.81 \%$ \\
\hline Opacity (ISO 2471): & $77.31 \%$ & $101.84 \%$ \\
\hline $\mathrm{pH}(1 \% \mathrm{w} / \mathrm{w}$ solution) (EN ISO \\
1262/1996)
\end{tabular}

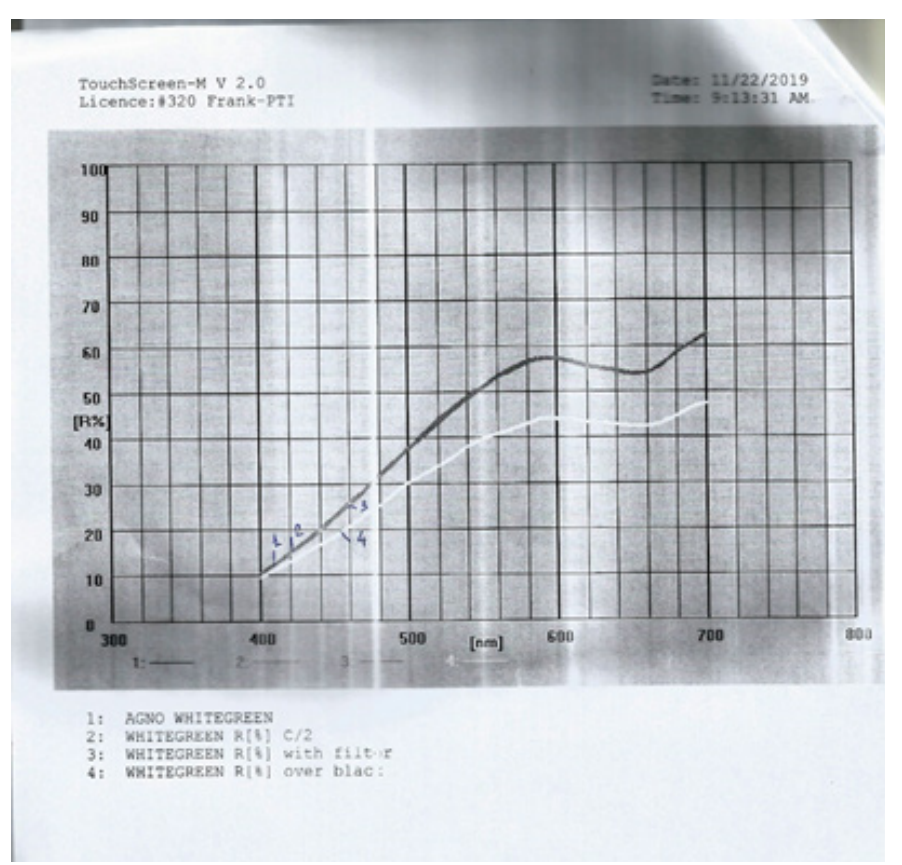

Figure 8: Reflectance graph for the green soap bar.

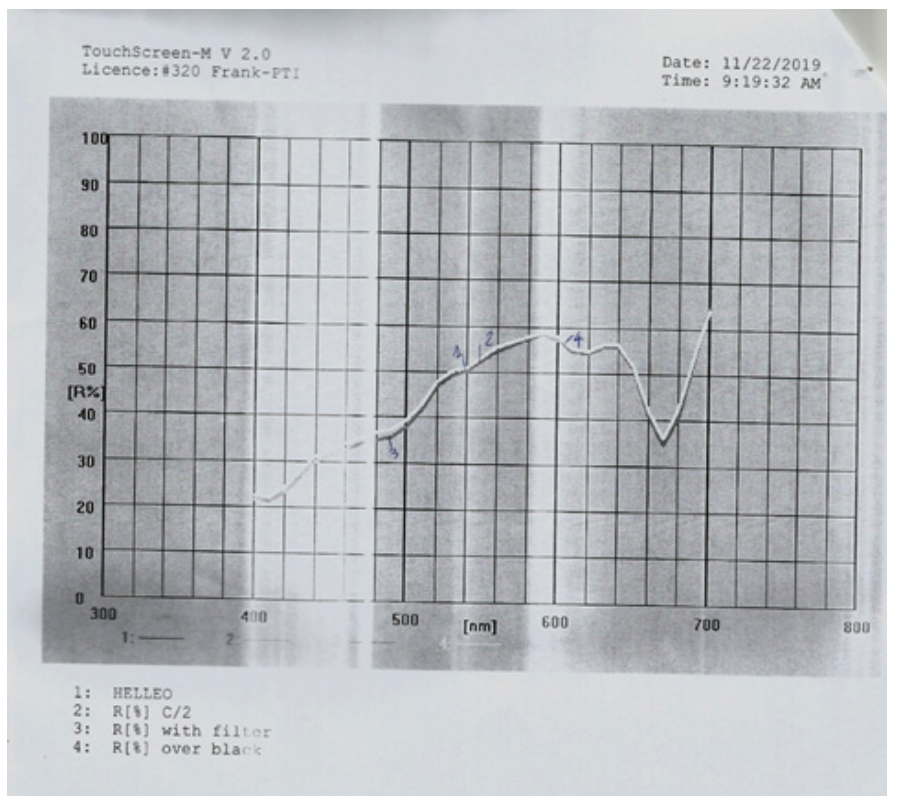

Figure 9: Reflectance graph for the white-green soap bar. 


\section{CIE $L^{*} a^{*} b^{*}$ values for the two soaps analyzed}

The color of the green soap in the CIE L*a*b* system for illuminant $\mathrm{C} / 2$ is $\mathrm{L}^{*}=76.07, \mathrm{a}^{*}=-3.96$ and $\mathrm{b}^{*}=33.99$. $\mathrm{L}$ is the measure of lightness and varies from 100 for a perfect white to 0 for the absolute black. Here -a indicates the greenness of the soap and $+b$ indicates its yellowness (Figure 8). The opacity calculated for the green soap bar is $77.31 \%$, and the transparency is $42.27 \%$. The color of the white soap in the CIE L*a*b* system for illuminant C/2 is $L^{*}=76.75, a^{*}=-3.01$ and $b^{*}=23.89$. $L$ is the measure of lightness and varies from 100 for a perfect white to 0 for the absolute black (Figure 9). Here -a indicates the greenness of the soap and $+b$ indicates its yellowness. Here the color measured indicates the presence of a green white, rather than a white soap. The opacity calculated for the second white-green soap bar is $101.84 \%$ and the transparency is $2.55 \%$.

\section{Conclusion}

The FTIR method and the Reflectance graph, are simple, accurate and easily applicable methods to the identification of finished products of anionic surfactants soap samples. Presently, we are investigating the use of FTIR for a wide variety of detergent, and cosmetic finished products.

\section{References}

1. Marie Sabo, John Gross, Ira E Rosenberg (1984) Quantitation of anionic surfactants in aqueous systems via Fourier transform infrared spectroscopy. J Soc Cosmet Chem 35(4): 207-220.

2. Jenkins JW, Kellenbach KO (1959) Identification of anionic surfaceactive agents by infrared absorption of barium salts. Anal Chem 31(6): 1056-1059.

3. ISO 685 (1975) 1975 analysis of soaps-determination of total alkali content and total fatty matter content $\left(1^{\text {st }}\right.$ edn). ISO (International Organization for Standardization).

4. ISO 673 (1981) 1981 soaps-determination of content of ethanolinsoluble matter $\left(2^{\text {nd }}\right.$ edn). ISO (International Organization for Standardization).

5. ISO 456 (1973) 1973 surface active agents-analysis of soapsdetermination of free caustic alkali $\left(1^{\text {st }}\right.$ edn). ISO (International Organization for Standardization). 